\title{
Topology Optimization of Rosette Eddy Current Array Sensor for Quantitative Monitoring Crack
}

\author{
Du Jin-qiang ${ }^{1, a, *}$, Chen Tao ${ }^{1, b}$, He Yu-ting ${ }^{1, c}$, Zhang Zhijun ${ }^{1, d}$, Li Peiyuan ${ }^{1, e}$ \\ ${ }^{1}$ Aeronautics and Astronautics Engineering College, Air Force Engineering University, Xi’an, China \\ ajq_du84@163.com, ${ }^{b} 762738050 @ q q . c o m,{ }^{c}$ hyt666@163.com, ${ }^{d}$ \\ supperzz8007@sina.con, ${ }^{\mathrm{a}}$ arthdaka@163.com
}

Keywords: topology optimization, rosette eddy current array sensor, finite element model, crack monitoring.

\begin{abstract}
In order to increase the crack monitoring sensitivity of a rosette eddy current array sensor, a crack-monitoring system was established, and an equivalent sensor model was developed by COMSOL. Then the influence of the sensor's topology on its sensitivity was investigated. The results indicate that the sense's sensitivity would reach the maximum when put the exciting and sensing windings in same plane. Increasing the horizontal space between exciting and sensing winding $\left(D_{H}\right)$ will enhance the sensor's sensitivity but reduce the sensor's output voltage. When increase the layer of winding, the output voltage will increase sharply and the sensor's sensitivity would decrease gradually. As the higher output voltage would facilitate the crack monitoring, there is a tradeoff between the sensor's output voltage and crack monitoring sensitivity in engineering application.
\end{abstract}

\section{Introduction}

Structural health monitoring (SHM) is the resident monitoring of structures/components by means of sensors integrated or applied to the structure. It is aimed at assuring the structural integrity of the aircraft, replacing on-event and periodic inspections to detect damages resulting from excessive loads, fatigue, corrosion, shocks...[1]. And SHM has become a research field of great interest for many years. The key to engineering application is the integration of the detection and damage identification techniques into powerful, cost-effective and robust systems.

Eddy current test method has been using for variety of applications, typically either as proximity sensors or for nondestructive evaluation in aviation, nuclear power plant, pressure vessel and so on [2]. The flexible eddy current sensor is a kind of eddy current sensor manufactured by flexible printed circuit board (FPCB) technics, which is shown in Fig.1[3,4]. Those senses are flexible, lightweight, and fit for SHM application. Hence, the flexible eddy current sensor is becoming a research field of great interest. JENTEK Sensors Inc. has developed the Meandering Winding Magnetometer (MWM) and the measurement grid inversion method, which has been used to detect and monitor fatigue cracks[5,6]. Alexi Rakow1 and Fu-Kuo Chang developed a structural health monitoring (SHM) fastener, which consisting of a conformable eddy current sensor film that is integrated with a metallic sleeve that goes around the fastener shank, for in situ monitoring of fatigue[3]. Ioannis Pitropakis and Helge Pfeiffer developed a flat coil sensor based on eddy current technology, for the purpose of structural health monitoring of critical aircraft components[7]. 


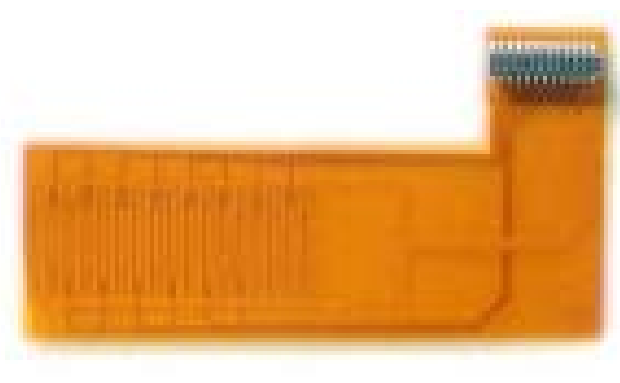

(a) MWM arrays

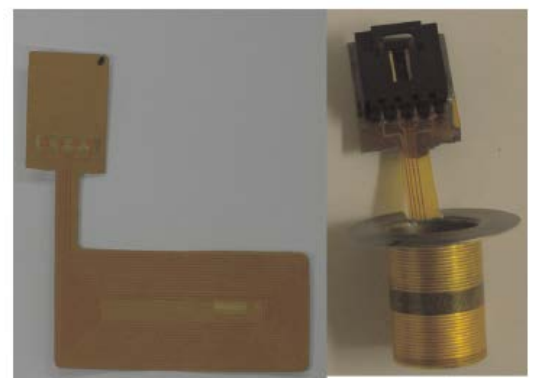

(b) AIME sensor

Figure1 Flexible eddy current sensor.

As flexible eddy current sensors are fabricated by FPCB technics, the turn of exciting coils and sensing coils are limited. Such as the MWM array sensor, the exciting coils and sensing coils are single-turn coils. As a result, output voltage of the sensor is faintness, just about several millivolts, which brought up great difficult for signal processing. Based on the segment monitoring method of the MWM array sensor, an equivalent finite-element model based on the rosette eddy current array sensor was established, and the topology of sensor are optimized to facilitate the crack monitoring.

\section{Rosette eddy current array sensor}

The rosette eddy current array sensor is used to monitor fatigue crack initiating and propagating at bolt holes. The geometry of rosette eddy current array sensor is shown schematically in Fig.2, where the cyclic exciting winding are periodically meandered and the sensing windings are distributed between the adjacent drive winding with equal spacing [8].

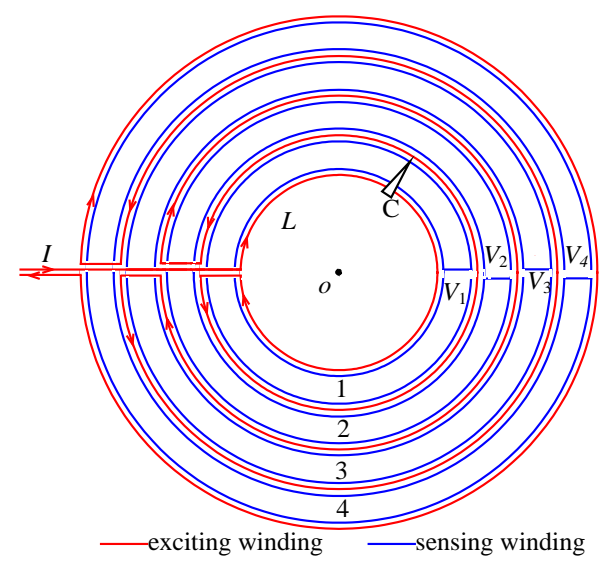

Figure2 Schematic of a rosette eddy current array sensor.

While applied to monitor fatigue cracks at edge of bolt hole, the sensor usually is integrated with structure as shown in Fig.3.The physical properties of the windings enable the sensor to quantitatively monitor crack damage. The source magnetic field is aroused by the exciting time-varying current I, and the reflected magnetic field is obtained from the sensing windings. When the sensor is integrated with the structure, crack damage will change the winding's output signal V. For example, As illustrated in Fig. 2, when crack damage (denoted by C) grows into the sense winding of channel 1 , the output signal $\mathrm{V}_{1}$ changes, while the other channel's output signals remain the same. By analyzing the output signal of each sensing winding, the length of crack can be quantified. 


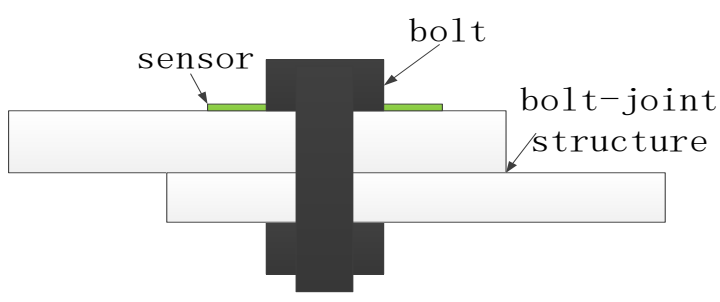

Figure3 Schematic of sensor integrated with structure.

\section{Equivalent finite element simulation model}

According to the symmetry and periodicity of the rosette eddy current array sensor, this paper establishes a one-channel winding model. An axisymmetric 2D geometry was adopted to simulate the model. A simplified physical model of the sensor while monitoring cracks is shown in Fig. 4.

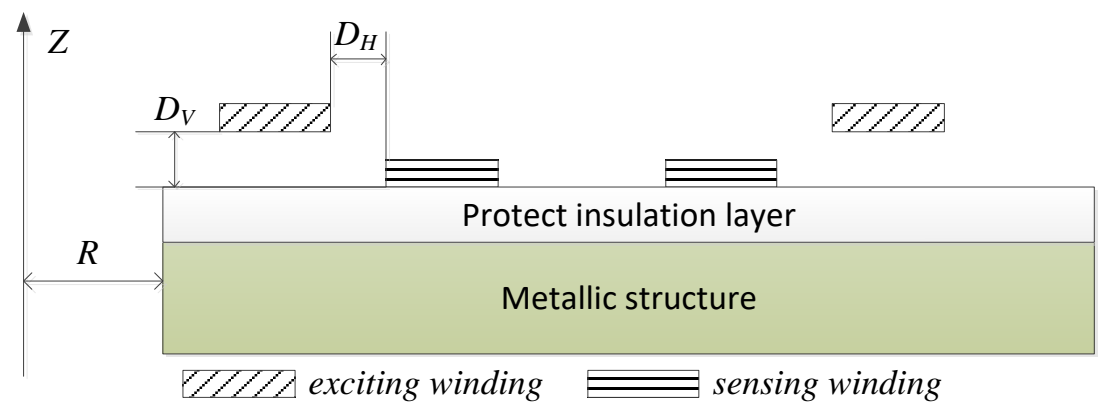

Figure4 Physical model of the sensor while monitoring cracks (cross section).

Here, $\mathrm{R}$ is the radius of bolt hole, $D_{V}$ is the vertical space between exciting and sensing winding, and $D_{H}$ is the horizontal space between exciting and sensing winding.

Fig. 5 shows the finite element model and material under test, which is established through the COMSOL AC/DC module. Material parameters in the model are listed in Table 1, whose relative permeability are all equal to 1 .

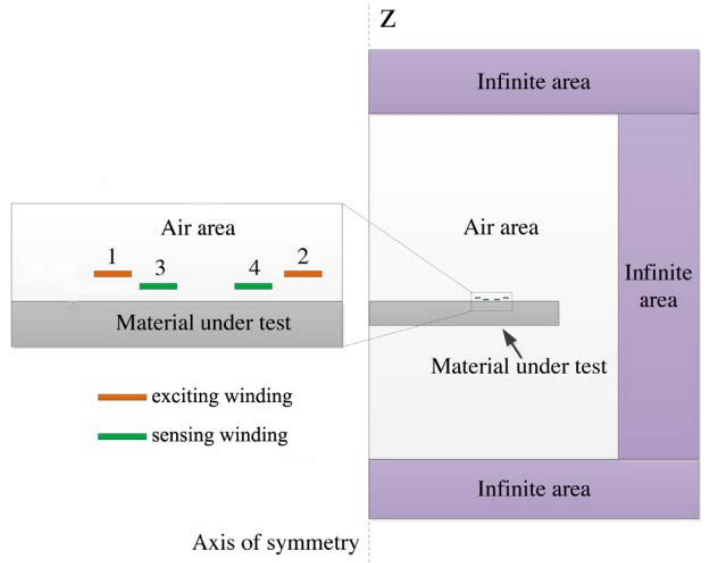

Figure5 Schematic of the finite element model.

Table 1 Material parameters.

\begin{tabular}{|c|c|c|}
\hline type & area & conductivity $(\mathrm{s} / \mathrm{m})$ \\
\hline air & air area & 0 \\
\hline copper & exciting and sensing winding & $5.998 \times 10^{7}$ \\
\hline 2A12-T4 aluminum alloy & Material under test & $1.74 \times 10^{7}$ \\
\hline
\end{tabular}

The described finite element model was applied to simulate crack monitoring of 2A12-T4 aluminum alloy structure. The veracity of the model was validate by experiment, the detail was presented in reference[9], the author is the member of our research team. 


\section{Topology optimization of the sensor}

\subsection{Define of the sensor's crack monitoring sensitivity}

According to the research of Goldfine[10], the crack damage of material under test could be equivalent to the change of the material's electromagnetic or geometric parameters. As 2A12-T4 aluminum alloy is a non-ferromagnetic material, the crack damage can be equivalent to the change of the material's conductivity in this paper. Thus, the conductivity sensitivity $S_{\sigma}$ of the model is a proxy to the monitoring's sensitivity in crack damage.

Here, the crack monitoring sensitivity $S_{\sigma}$ is defined as

$$
S_{\sigma}=\sqrt{\left(\frac{\frac{\Delta z_{R}}{z_{R}^{0}}}{\frac{\Delta \sigma}{\sigma_{0}}}\right)^{2}+\left(\frac{\frac{\Delta z_{\theta}}{z_{\theta}^{0}}}{\frac{\Delta \sigma}{\sigma_{0}}}\right)^{2}}
$$

Where $Z_{R}$ is the amplitude of sensor trans-impedance, $Z_{\theta}$ is the phase of sensor trans-impedance, and $\sigma$ is the conductivity of the material under test.

\subsection{Topology optimization of the sensor}

The sensor was applied to monitor the crack in 2A12-T4 aluminum alloy structure. The effect of $D_{V}, D_{H}$, and layers of windings on the sensor's crack monitoring sensitivity $S_{\sigma}$ is investigated by the simulation model.

(1) Vertical space between exciting and sensing winding $\left(D_{V}\right)$

The relationship between $S_{\sigma}$ and $D_{V}$ is shown in Fig.6. As the vertical space between exciting and sensing winding $D_{V}$ increasing, the sensitivity $S_{\sigma}$ decreases greatly. When put the exciting and sensing windings in same plane, the $S_{\sigma}$ will reach the maximum.

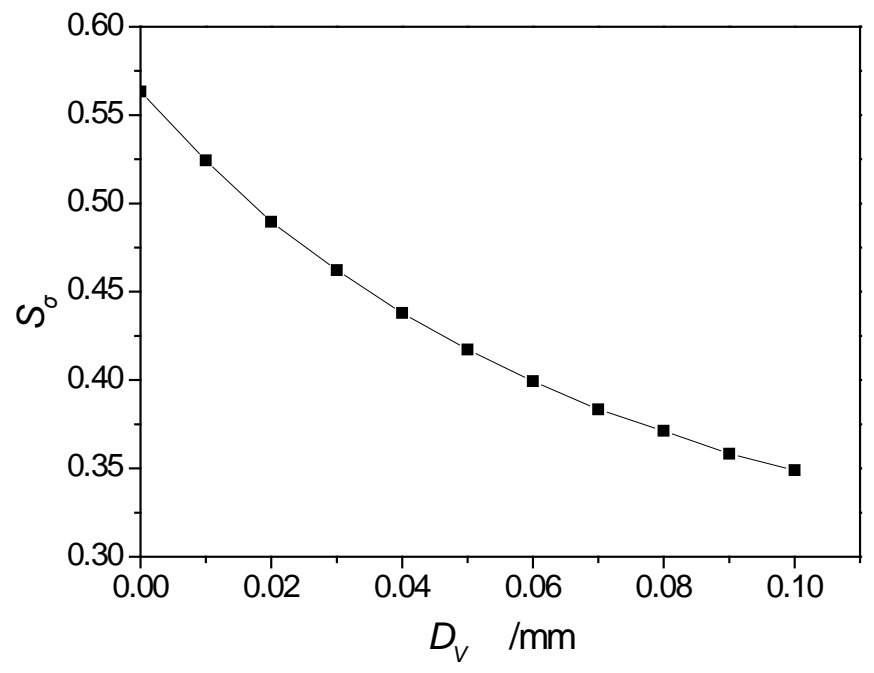

Figure6 crack monitoring sensitivity $S_{\sigma}$ varying by $D_{V}$.

(2) Horizontal space between exciting and sensing winding $\left(D_{H}\right)$

Fig.7 reveals that the influence of $D_{H}$ on the sensor's sensitivity $S_{\sigma}$ and output voltage. Along with the increasing of horizontal space between exciting and sensing winding, the sensor sensitivity $S_{\sigma}$ increases greatly, but the sense's output voltage decreases sharply, the minimum voltage is just about $10 \mathrm{mv}$, which would brought up some difficulty in engineering application. Therefore, there should be a tradeoff between $S_{\sigma}$ and output voltage. 


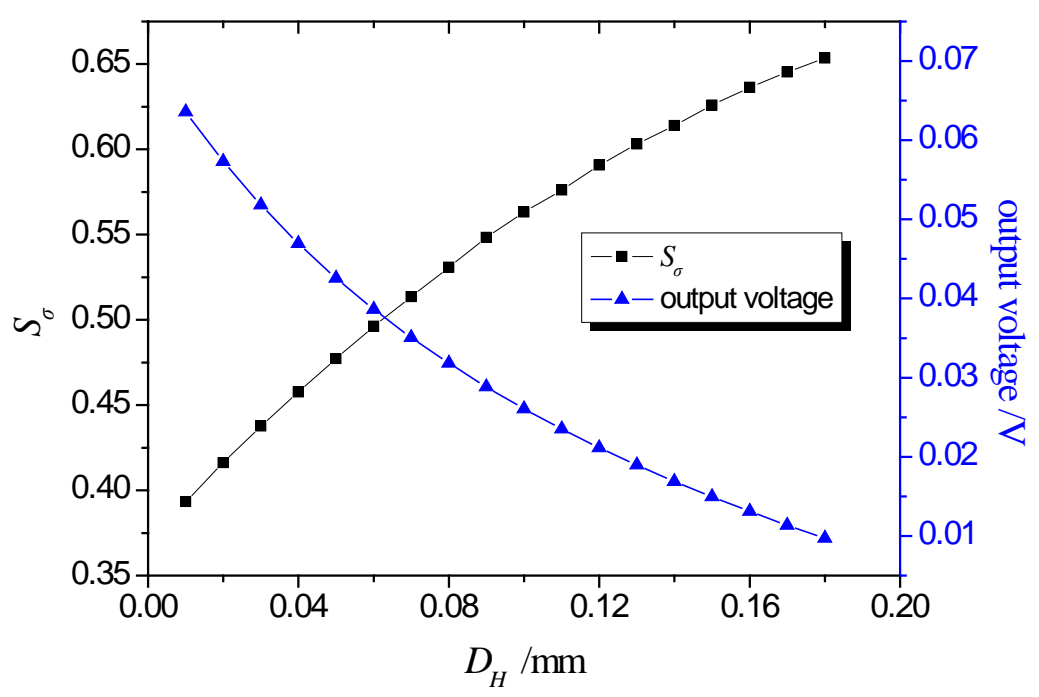

Figure7 Output voltage and $S_{\sigma}$ varying by $D_{H}$.

(3) Multi-layers winding

As the origin rosette eddy current array sensor only has a single-turn exciting and sensing winding, the output signal is just dozens of millivolt, which would easily be disturbed by noise. The way to improve this problem is increase the turn of windings. In flexible printed circuit board technics, this means increase the layer of windings.

Firstly, a simulation model of rosette eddy current array sensor with two layers of winding was established by FEM method. The detail of windings is shown in Fig.8, and $D_{B}$ is the distance between two layers.

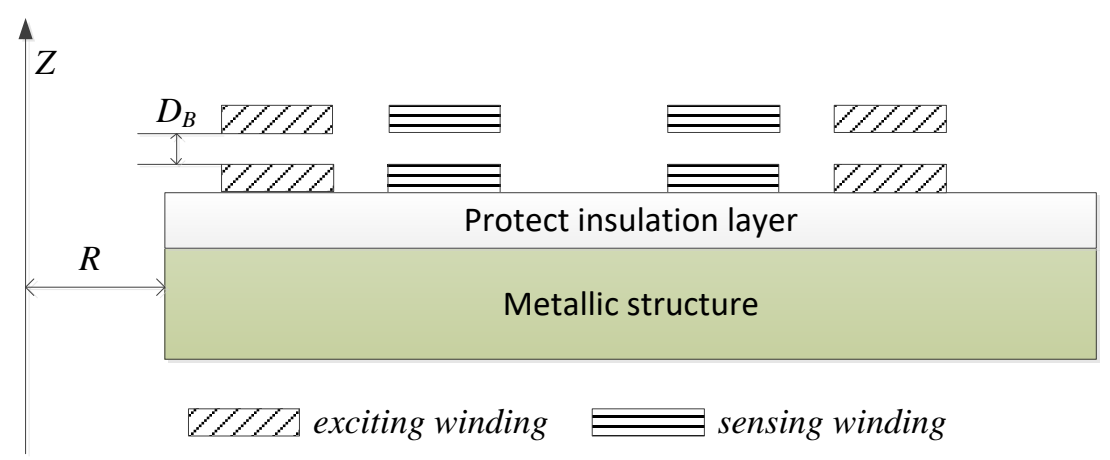

Figure8 Physical model of the sensor with two layers of winding.

The effect of distance between two layers $D_{B}$ on the sensor's output voltage and sensitivity is shown in Fig.9. Compared Fig.9(a) with Fig.7, it is clear that the output voltage increases almost twice. As the distance between layers increased, the output voltage increased and the sensor's sensitivity decreased gradually. 


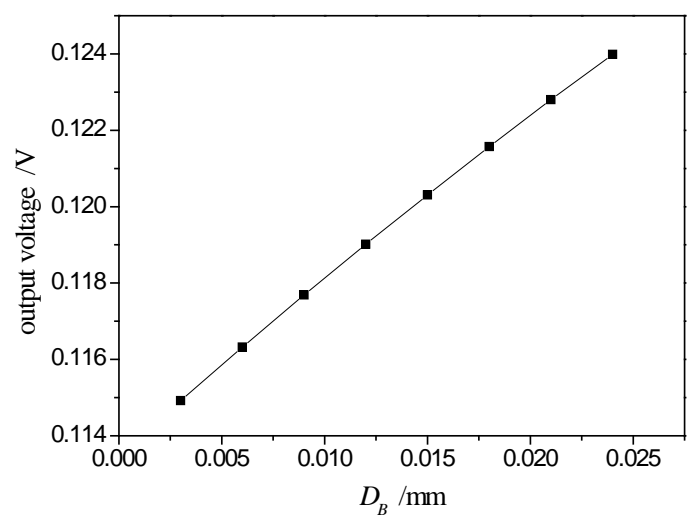

(a) Output voltage vs. $D_{B}$

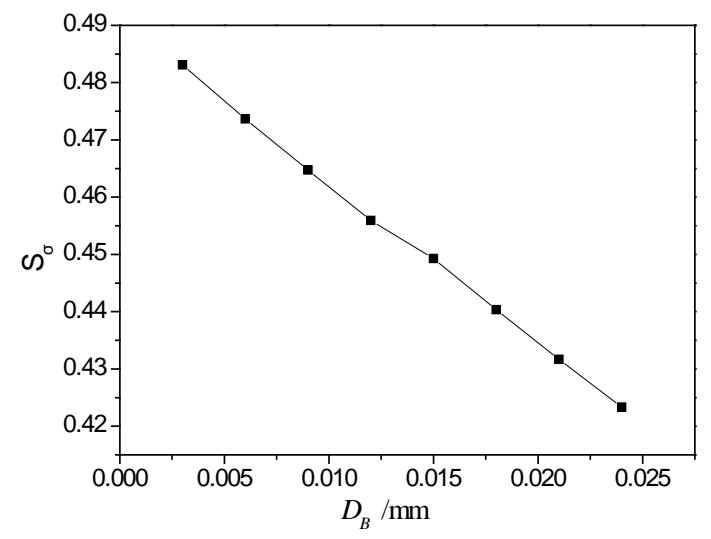

(b) $S_{\sigma}$ vs. $D_{B}$

Figure9 Output voltage and $S_{\sigma}$ varying by $D_{B}$.

Furthermore, a simulation model of rosette eddy current array sensor with three layers of winding was also investigated, and the distances between layers $\left(D_{B}\right)$ are same. The influence of distance between layers $\left(D_{B}\right)$ on the sensor's output voltage and sensitivity $S_{\sigma}$ is plotted in Fig.10.

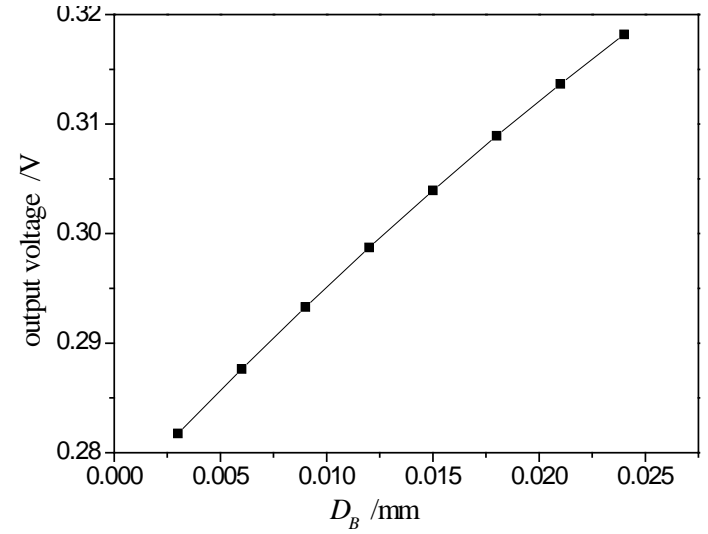

(a) Output voltage vs. $D_{B}$

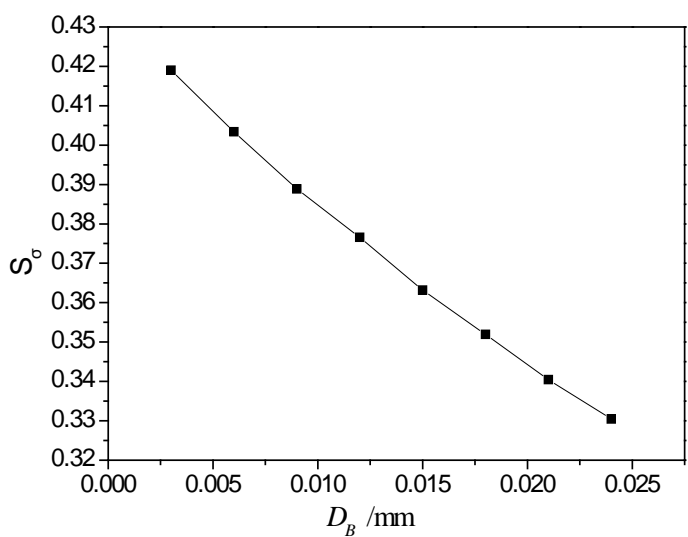

(b) $S_{\sigma}$ vs. $D_{B}$

Figure10 Output voltage and $S_{\sigma}$ varying by $D_{B}$.

It is shown that Fig.10 and Fig.9 have the same trend as the distance between layers $D_{B}$ increasing. Compared with two layer sensor, the output voltage increased more than twice and the sensor's sensitivity is much lower.

\section{Conclusions}

In this paper, an equivalent finite element model of rosette eddy current array sensor was established, and the influence of the sensor's topology on its sensitivity was investigated. It is shown that

(1) When put exciting and sensing windings in same plane, the sensor will get the optimum sensitivity in crack monitoring.

(2) Increasing the horizontal space between exciting and sensing winding would enhance the sensor's sensitivity but reduce the sensor's output voltage. The minimum voltage is just about $10 \mathrm{mv}$, which would be easily disturbed by noise. Therefore, there should be a tradeoff between $S_{\sigma}$ and output voltage in choosing $D_{H}$.

(3)When increasing the layers of winding, the output voltage will increase sharply, and the sensor's sensitivity would decrease gradually. As the higher output voltage would facilitate the crack monitoring, there is a tradeoff between the sensor's output voltage and crack monitoring sensitivity in engineering application. 


\section{Acknowledgements}

The work is supported by the National Nature Science Foundation of China (Project No. 51507186), Shaanxi Nature Science Foundation (Project No. 2016JQ5004), and China Postdoctoral Science Foundation (Project No. 2016M592967)

\section{References}

[1] Herman Vander Auweraer, Bart Peeters. (2003) Sensors and systems for structural health monitoring. Journal of Structural Control, 10, 117-125.

[2] Fu Xiaoqiang, Wu Sujun, Zhang Jiajia. (2009) Application of eddy current sensor array non-destructive testing technology on large passenger aircraft. Civil Aircraft Design and Research, 84-88.

[3] Alexi Rakow, Fu-Kuo Chang. (2011) A structural health monitoring fastener for tracking fatigue crack growth in bolted metallic joints. Structural Health Monitoring, 11(3), 253-267.

[4] V. Zilbersteina, D. Grundya, V. Weissa, et al. (2005) Early detection and monitoring of fatigue in high strength steels with MWM-Arrays. International Journal of Fatigue, 27, 1644-1652.

[5] V. Zilberstein, D. Grundy, V. Weiss, et al, (2005) Early detection and monitoring of fatigue in high strength steels with MWM-arrays, Int. J. Fatigue, 25 (10), 1644-1652.

[6] N. Goldfine, D. Grundy, C. Craven, et al. Virginia Beach, USA, (2007) Damage and usage monitoring for vertical flight vehicles, American Helicopter Society 63rd Annual Forum, 8217.

[7] Ioannis Pitropakis, Helge Pfeiffer, Martine Wevers. (2012) Crack detection in aluminum plates for aerospace applications by electromagnetic impedance spectroscopy using flat coil sensors. Sensors and Actuators A: Physical, 176, 57-63.

[8] Ding Hua, He Yuting, Jiao Shenbo, et al. (2012) Fatigue crack monitoring of metallic structures based on eddy current array sensor, J. Beijing Univ. Aeronaut. Astronautics, 38 (12), 1629-1633.

[9] Peiyuan Li, Li Cheng, Yuting He, et al. (2016) Sensitivity boost of rosette eddy current array sensor for quantitative monitoring crack. Sensors and Actuators A:Physical, 246, 129-139.

[10] N. Goldfine, V. Zilberstein, A. Washabaugh, et al. (2003) Eddy current sensor networks for aircraft fatigue monitoring, Mater. Eval, 61(7), 852-859. 